Rev. Biol. Trop., 47(4): 923-928, 1999

www.ucr.ac.cr www.ots.ac.cr www.ots.duke.edu

\title{
Anomuros (Anomura) del arrecife de Cabo Pulmo-Los Frailes y alrededores, Golfo de California
}

\author{
María del Socorro García-Madrigal
}

Consultoría e Investigación Ambiental, A.C. (CINAM), Apdo. Postal 466, Chetumal, QROO, 77000, México. Nueva dirección/new address: El Colegio de la Frontera Sur (ECOSUR), Apdo. Postal 424, Chetumal, QROO, 77000, México. Fax: (983) 204-47; e-mail: ms_garcia_m@hotmail.com

Recibido 20-X-1998. Corregido 9-VII-1999. Aceptado 3-VIII-1999

\begin{abstract}
During 1988-1989 seven expeditions were made in the Cabo Pulmo-Los Frailes reef and fifty-seven anomurans were collected in fragments of Pocillopora elegans (nine species of Porcellanidae and one of Diogenidae). Twenty-two species were recorded previously for the study area and three are new records, all distributed in four families. The Porcellanidae had most species (15), followed by the Diogenidae (7), Hippidae (2) and the Coenobitidae (1). Most abundant were Megalobrachium tuberculipes (20 specimens), Petrolisthes edwardsii (nine specimens), and $P$. hirtispinosus (seven specimens). All species are recognized as members of the Eastern Tropical Pacific fauna, although Hippa pacifica is widespread in the Indopacific. Four species are restricted to the Cortez province (16\%). Anomurans collected in these reefs appear to represent $21 \%$ of the total number of species recorded once for the area of the Gulf of California, Nayarit, Sinaloa, Western Coast of Baja Califormia Sur and economic exclusive zone of this states.
\end{abstract}

Key words: Coral reef, faunistic, hermit crabs, mole crabs, Pocillopora, porcellanid crabs.

Los anomuros son un grupo que ha sido estudiado de forma heterogénea en la costas pacíficas del continente americano (Brusca 1980) y no hay síntesis relevantes recientes (Hendrickx 1993). En algunos grupos (e.g. Isocheles, Pagurus, entre otros) el estudio incompleto ha generado confusión, a pesar de las revisiones de algunas especies del Pacífico oriental (Haig y Harvey 1991, Haig y McLaughlin 1991, Harvey y McLaughlin 1991 y McLaughlin y Haig 1993).

Debido al efecto de las corrientes frías de California y de Perú/Humboldt, la franja subtropical-tropical del Pacífico es más angosta que en otras regiones del mundo (ver Hendrickx 1995). Consecuentemente, los anomuros del Pacífico americano se caracterizan por tener ámbitos de distribución muy estrechos, aunque algunas formas se distribuyen ampliamente alcanzando las zonas templadas y frías
(Haig et al. 1970, Carvacho 1980). Excepcionalmente se presentan formas bipolares, anfiamericanas e indopacíficas (Haig 1968, Brusca 1980, Hendrickx 1993).

Para el arrecife de Cabo Pulmo-Los Frailes, se cuenta con cinco trabajos publicados anteriormente (Cuadro 1), que mencionan a los anomuros: Glassell (1937) registró cuatro especies de diogénidos para la zona; Steinbeck y Ricketts (1941) añadieron cuatro especies; por su parte Haig (1960) informó de seis especies; Brusca y Thomson (1977) registraron otras cinco; y por último Brusca (1980) informó de tres especies más.

El presente trabajo incluye los resultados del análisis de la riqueza y biogeografía de las especies de anomuros del arrecife de Cabo Pulmo-Los Frailes y alrededores, tanto en lo que se refiere a los registros previos como los obtenidos por los muestreos recientes. 


\section{CUADRO 1}

Listad• de especies de anomuros del arrecife de Cabo Pulmo-Los Frailes y alrededores, hábitats de recolección (en Cabo Pulmo) y composición zoogeográfica.

Checklist of anomuran species from Cabo-Pulmo-Los Frailes reef and vicinity, collection habitats (in Cabo Pulmo) and zoogeographic composition

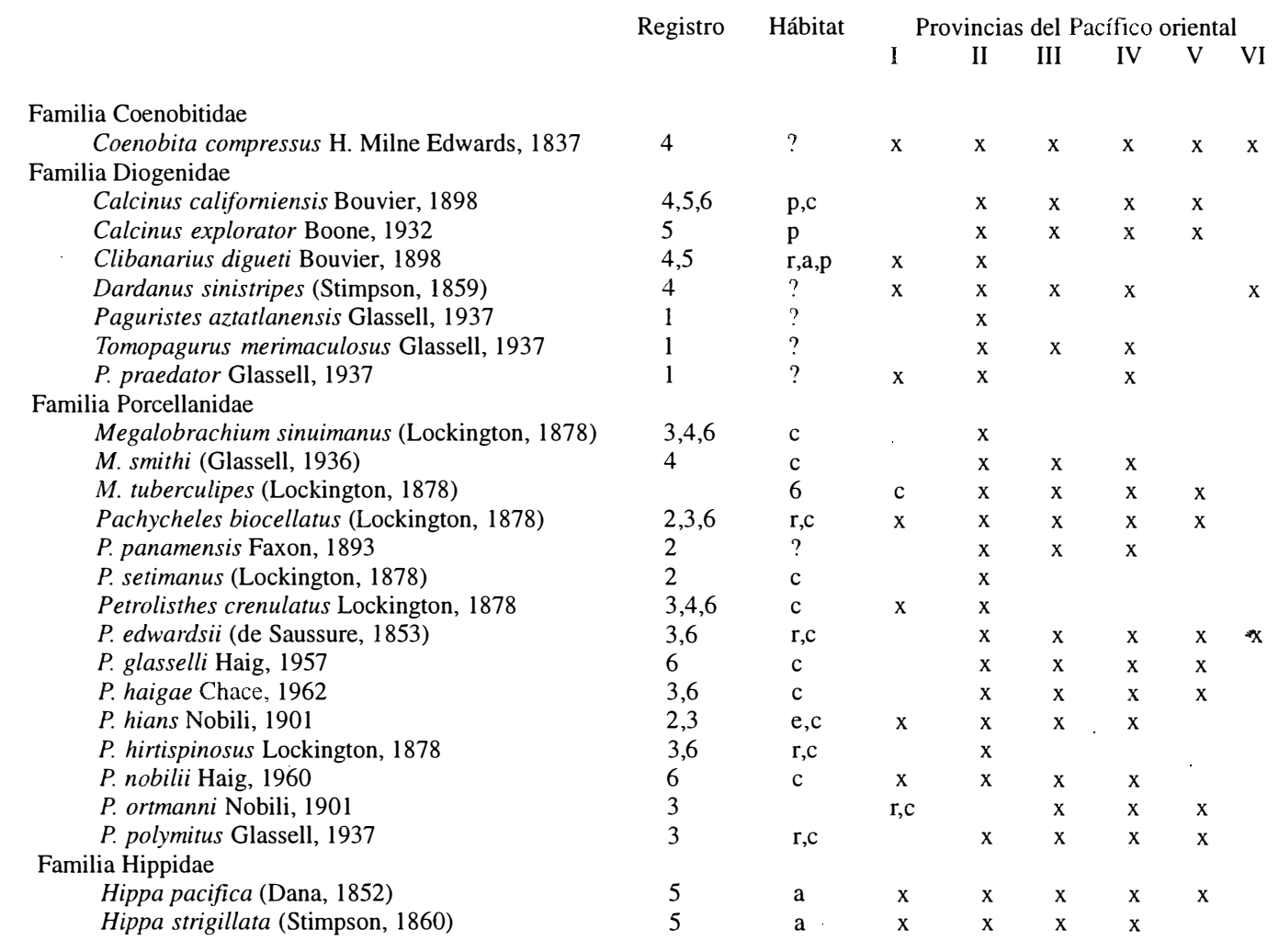

Referencias: 1) Glassell 1937, 2) Steinbeck y Ricketts 1941, 3) Haig 1960, 4) Brusca y Thomson 1977, 5) Brusca 1980, 6) este trabajo. Hábitat donde han sido recolectados: $c=$ coral, $r=$ roca, $a=$ playa arenosa, $e=$ epifauna, $p=p o z o s$ de marea. Provincias del Pacífico oriental: $\mathrm{I}=$ transición, $\mathrm{II}=$ cortesiana, $\mathrm{III}=$ mexicana, $\mathrm{IV}=$ panámica, V= Galápagos, VI= peruana.

\section{MATERIALES Y MÉTODOS}

El método y la descripción del área de estudio se detallaron en un trabajo previo (García-Madrigal y Bastida-Zavala 1999). La identificación de las especies de anomuros se realizó con ayuda de literatura especializada (Haig 1960, Chace 1962, Haig et al. 1970 y Brusca 1980). Todos los ejemplares se catalogaron y depositaron en la Colección Enrique Rioja de Invertebrados Acuáticos de Consultoría e Investigación Ambiental (CINAM) en Chetumal, Quintana Roo.
La información actualizada relacionada con la distribución de los anomuros se obtuvo de diversas fuentes (Haig et al. 1970, Gore 1982, Hendrickx 1992, Moran y Dittel 1993). Para el análisis zoogeográfico se siguió el esquema de provincias del Pacífico oriental propuesto por Brusca y Wallerstein (1979): Zona de transición (de Punta Eugenia a Cabo San Lucas), Provincia cortesiana (todo el golfo de California), Provincia mexicana (de Cabo San Lucas a Tangolunda o bahía Tangola-Tangola, Oaxaca), Provincia panámica .(de Tangolunda o bahía Tangola-Tangola, Oaxaca, a Ecuador), Provincia Galápagos (sólo las islas Galápagos), Provincia peruana (Perú/Chile). 
Para efectos de comparación se utiliza el área II del Pacífico mexicano, región con 704 $200 \mathrm{~km}^{2}$ de piso oceánico que comprende el golfo de California, las áreas costeras de Baja California Sur, Nayarit y Sinaloa además de su zona económica exclusiva (200 millas náuticas) presentada por Hendrickx (1993).

\section{RESULTADOS}

Las muestras contenían 57 ejemplares de porcelánidos, asignados a nueve especies, de las cuales tres son nuevos registros para el arrecife de Cabo Pulmo-Los Frailes: Megalobrachium tuberculipes, Petrolisthes glasselli y $P$. nobilii (Cuadro 1). Las especies más abundantes en las muestras fueron el M. tuberculipes (20 ejemplares), P. edwardsii (9), P. hirtispinosus (7), P. haigae y Pachycheles biocellatus (6 ejemplares cada una).

Por otra parte, el número de especies de anomuros registrados en la literatura para el área de estudio era de 22 especies, repartidas en cuatro familias. Con las especies registradas en este trabajo, el número de anomuros para el arrecife de Cabo Pulmo-Los Frailes y alrededores se incrementó a 25 especies repartidas en el mismo número de familias. Las familias Porcellanidae y Diogenidae se mantienen como dominantes con 15 y siete especies, respectivamente. (Cuadro 1).

Se tienen 19 registros del hábitat en los anomuros del arrecife de Cabo Pulmo-Los Frailes y alrededores (Cuadro 1), dominando el coral (53.6\%), seguido por los fondos rocosos $(21.4 \%)$, los pozos de marea (10.7\%), la playa arenosa $(10.7 \%)$ y por último los epibiontes (3.6\%).

\section{DISCUSIÓN}

Faunística y notas ecológicas: Como las muestras no tienen igual volumen no pueden hacerse comparaciones de abundancia relativa (cf. García-Madrigal y Bastida-Zavala 1999), salvo un análisis directo de la riqueza específica. El Coral Los Frailes presenta la mayor ri- queza de anomuros (diez especies), seguida por la tercera barra arrecifal (nueve especies) y por la segunda barra arrecifal (seis especies), mientras que ningún anomuro estuvo representado en la primer barra arrecifal; sin embargo, estas diferencias probablemente son reflejo del esfuerzo de muestreo y/o el volumen de los fragmentos extraídos.

Comparando la riqueza específica de anomuros con otras regiones (Cuadro 2), se observa que la riqueza de especies en el arrecife de Cabo Pulmo-Los Frailes y sus alrededores es modesta, por ejemplo en relación con el área II del Pacífico mexicano (sensu Hendrickx 1993) o el atolón Enewetak. Sin duda, la intensidad y tiempo de las campañas de muestreo en estos sitios, así como el área y hábitats contemplados, permitieron la obtención de un mayor número de especies. Sin embargo, si se compara la riqueza específica de anomuros en relación con otras localidades con mayor superficie cubierta, como el Caribe mexicano, bahía Magdalena, bahía Maruata, isla Gorgona o las especies encontradas en corales pocilopóridos en Panamá, se observa que el arrecife de Cabo Pulmo-Los Frailes está muy bien representado. No obstante, es posible que el número de especies de anomuros en este arrecife se incremente con un programa de muestreo más intenso y detallado.

Aun así, la riqueza específica de los anomuros de este pequeño arrecife y sus alrededores (menos de $30 \mathrm{~km}^{2}$ ), representa el $21 \%$ de las 119 especies registradas en el área II del Pacífico mexicano; esta proporción es equivalente a la de los braquiuros de esta misma zona (García-Madrigal y Bastida-Zavala 1999).

La elevada riqueza de anomuros (principalmente porcelánidos) se debe a la heterogeneidad de sustratos que ofrece el arrecife, tanto para los anomuros como para otros invertebrados. De las 90 especies de porcelánidos registradas para el Pacífico americano (Carvacho 1980) el $16.6 \%$ está presente en Cabo PulmoLos Frailes.

Haig (1960) destaca el hábito comensal de los porcelánidos e incluye los diferentes hospederos, entre los que se encuentran esponjas, equinodermos, corales gorgónidos y madrepó- 
ridos, además de anémonas, cangrejos ermitaños y poliquetos. La especificidad es alta y los arrecifes son hábitats importantes para los porcelánidos; de las 36 especies recolectadas en las expediciones del velero ZACA en el Pacífico oriental, 18 (50\%) fueron recolectados en arrecifes coralinos (Haig 1968).

Petrolisthes glasselli, P. haigae, P. hians, P. polymitus y Pachycheles biocellatus son tí- picas del coral, siendo muy raro encontrarlos sobre cualquier otro sustrato (Haig 1960, 1968), aunque en este trabajo Petrolisthes hians y $P$. polymitus no se encontraron en el área de estudio; los géneros Calcinus y Clibanarius se encontraron en pozas de marea, además Calcinus también se encuentra en coral. El género Hippa se asocia con las playas arenosas (Cuadro 1).

\title{
CUADRO 2
}

\begin{abstract}
Comparación entre diferentes trabajos de la riqueza específica y composición zoogeográfica de los anomuros del arrecife de Cabo Pulmo-Los Frailes y alrededores

Comparative between different works of the species richness and zoogeographic composition of the anomurans from Cabo Pulmo-Los Frailes reef and vicinity
\end{abstract}

Comparación de la riqueza específica entre varios sitios.

Referencia

Hendrickx (1993)

Markham et al. (1990)

Werding \& Haig (1983)

Abele (1976)

Garth et al. (1987)

Haig et al. (1970)

Hernández (1995)

Este trabajo
Sitio

área II del Pacífico de México (ver texto)

Caribe mexicano (Quintana Roo)

isla Gorgona (Colombia)

Panamá

atolón Enewetak e islas Marshall (Pacífico central)

bahía Magdalena

bahía Maruata (Michoacán)

Cabo Pulmo-Los Frailes y sus alrededores
Especies incluidas

$119^{b}$
$26^{b}$
$18^{c}$
$13^{b, d}$
$76^{b}$
$32^{b}$
$10^{c}$
$25^{b}$

${ }^{a}$ El arreglo es de mayor a menor superficie contemplada, ${ }^{b}$ todas las especies de anomuros registradas para estas zonas, ${ }^{c}$ sólo porcelánidos, d sólo las especies de anomuros encontrados en Pocillopora damicornis en el Pacífico de Panamá.

Comparación zoogeográfica.

\begin{tabular}{|c|c|c|c|c|c|c|c|c|}
\hline \multirow[b]{2}{*}{ Referencia } & \multirow[b]{2}{*}{ Sitio } & \multicolumn{6}{|c|}{ Provincias del Pacífico oriental (\%) } & \multirow{2}{*}{$\begin{array}{l}\text { Especies } \\
\text { incluidas }\end{array}$} \\
\hline & & I & II & III & IV & $\mathrm{V}$ & VI & \\
\hline Haig et al. (1970) a & bahía Magdalena & 100 & 75 & 15.6 & 46.2 & 12.5 & 9.37 & 32 \\
\hline Haig et al. (1970) b & bahía Magdalena & 100 & 75 & 66.6 & 0 & 0 & 0 & 12 \\
\hline Hernández (1995) a & bahía Maruata & 50 & 90 & 100 & 100 & 30 & 10 & 10 \\
\hline Este trabajo ${ }^{a}$ & Cabo Pulmo-Los Frailes & 52 & 100 & 72 & 76 & 32 & 8 & 25 \\
\hline Este trabajo ${ }^{b}$ & Cabo Pulmo-Los Frailes & 53 & 100 & 86.6 & 86.6 & 40 & 0 & 15 \\
\hline
\end{tabular}

a anomuros, b sólo porcelánidos. Provincias del Pacífico oriental: I= transición, II= cortesiana, III= mexicana, IV= panámica, $\mathrm{V}=$ Galápagos, $\mathrm{VI}=$ peruana.

Análisis zoogeográfico: Sólo una especie, Hippa pacifica, tiene amplia distribución y ha sido encontrada también en la región Indopacífica. De la totalidad de especies registradas, 18 se extienden en la Provincia mexicana y 19 hasta la Provincia panámica.

De las especies restringidas al Pacífico oriental, cuatro (16\%) son especies endémicas de la Provincia cortesiana: el diogénido Paguristes aztatlanensis (que hasta el momento só- lo se ha encontrado en Cabo Pulmo), así como los porcelánidos Megalobranchium sinuimanus, Pachycheles setimanus y Petrolisthes hirtispinosus (Cuadro 1).

La fauna de anomuros del arrecife de Cabo Pumo-Los Frailes es netamente tropical, restringiéndose a las provincias cortesiana, mexicana y panámica (Cuadro 2). En cambio los anomuros de bahía Magdalena (Haig et al. 1970) tienen afinidad subtropical. Esto confirma una vez 
más que la distribución de anomuros es más restringida que la de braquiuros (García-Madrigal y Bastida-Zavala 1999).

Asimismo, considerando sólo a los porcelánidos del arrecife, se repite básicamente el mismo esquema anterior (Cuadro 2); mientras que los porcelánidos de bahía Magdalena tienen una afinidad subtropical más marcada, distribuyéndose solo en las provincias de transición, cortesiana y mexicana (Haig et al. 1970). Por su parte, los porcelánidos de bahía de Maruata están mucho mejor representados en las provincias mexicana y panámica (Hernández 1995).

Los porcelánidos son estenotérmicos (Carvacho 1980) de ahí que presenten distribuciones más restringidas que los braquiuros, confirmando el esquema propuesto por Brusca y Wallerstein (1979), tanto para los anomuros en general, como de los porcelánidos en particular.

Los anomuros del arrecife de Cabo Pulmo-Los Frailes presentan una riqueza especifica elevada, además se comprueba que el arrecife es muy importante como hábitat para estos organismos. Se recomienda realizar estudios más intensivos para un mejor manejo de este ecosistema.

\section{AGRADECIMIENTOS}

Se agradece a Patricia Hernández-Cortés, James Ketchum, Rodolfo Moreno, Héctor Reyes-Bonilla (UABCS, La Paz) y Rolando Bastida-Zavala (CINAM), por la obtención de las muestras. La revisión y comentarios de José Rolando Bastida-Zavala y Sergio Salazar-Vallejo (ECOSUR, Chetumal) y los comentarios de dos árbitros anónimos, mejoraron la calidad del trabajo.

\section{RESUMEN}

El análisis de los fragmentos del coral Pocillopora elegans recolectados en siete campañas de muestreo durante 1988 y 1989 resultó en 57 ejemplares de anomuros, identificándose nueve especies de la familia Porcellanidae y una especie de Diogenidae, de los cuales tres especies son nuevos registros para el área. Para la zona había 22 especies de anomuros registradas; ahora hay 25 especies repartidas en cuatro familias. Porcellanidae contiene el mayor número de especies (15), seguida por Diogenidae (7), Hippidae (2) y Coenobitidae (1). Las especies más abundantes fueron $\mathrm{Me}$ galobrachium tuberculipes (20 ejemplares), Petrolisthes edwardsii (9) y P. hirtispinosus (7). Sólo Hippa pacifica (4\%) presenta distribución Indopacífica, el resto se distribuye en áreas más restringidas del Pacífico oriental tropical y subtropical. Cuatro especies son endémicas de la provincia cortesiana (16\%). Las especies de anomuros de este arrecife y sus alrededores representan el $21 \%$ de todas las especies registradas para la región del golfo de California, costa occidental de Baja California Sur, áreas costeras de Nayarit, Sinaloa y zona económica exclusiva de estos estados.

\section{REFERENCIAS}

Abele, L.G. 1976. Comparative species composition and relative abundance of decapod crustaceans in marine habitats of Panama. Mar. Biol. 38: 263-278.

Brusca, R.C. 1980. Common Intertidal Invertebrates of the Gulf of California. Arizona, Tucson, 513 p.

Brusca, R.C. \& D.A. Thomson. 1977. Pulmo Reef: The only "coral reef" in the Gulf of California. Cienc. Mar. (1975) 2: 37-53.

Brusca, R.C. \& B.R. Wallerstein. 1979. Zoogeographic patterns of idoteid isopods in the Northeast Pacific, with a review of shallow water zoogeography of the area. Bull. Biol. Soc. Wash. 3: 67-105.

Carvacho, A. 1980. Los porcelánidos del Pacífico americano: un análisis biogeográfico (Crustacea: Decapoda). An. Centr. Cienc. Mar Limnol. UNAM 7: 249-258.

Chace, F.A., Jr. 1962. The non-brachyuran decapod crustaceans of Clipperton Island. Proc. U.S. Natl. Mus. 113: 605-635.

García-Madrigal, M.S. \& J.R. Bastida-Zavala. 1999. Cangrejos braquiuros (Brachyura) del arrecife de Cabo Pulmo-Los Frailes y alrededores, Golfo de California, México. Rev. Biol. Trop. 47: 119-128.

Garth, J.S., J. Haig \& J.W. Knudsen. 1987. Crustacea Decapoda (Brachyura and Anomura) of Enewetak atoll, pp: 235-261. In D.M. Devaney, E.E. Reese, B.L. Burch \& P. Helfrich (eds.). The Natural History of Enewetak Atoll. Vol. 2. Biogeography and Systematics. U.S. Dep. Energy, Off. Scient. Techn. Inform. Tennessee.

Glassell, 1937. The Templeton Croker Expedition, XI. Hermit crabs from the Gulf of California and the 
west coast of Lower California. Zoologica, NY 22: 241-263.

Gore, R.H. 1982. Porcellanid crabs from the coasts of Mexico and Central America (Crustacea: Decapoda: Anomura). Smithson. Contr. Zool. 363: 1-34.

Haig, J. 1960. The Porcellanidae (Crustacea Anomura) of the Eastern Pacific. Allan Hancock Pac. Exped. 24: $1-440$.

Haig, J. 1968. Eastern Pacific Expeditions of the New York Zoological Society. Porcellanid crabs (Crustacea: Anomura) from the West Coast of Tropical America. Zoologica, NY 53: 57-74.

Haig J. \& A.W. Harvey. 1991. Three new species of the Pagurus lepidus complex (Decapoda, Anomura, Paguridae) from the eastern Pacific. Contr. Sci. 430: 111.

Haig J. \& P.A. McLaughlin. 1991. Identity of Pagurus lepidus (Decapoda, Anomura, Paguridae) and description of a new eastern Pacific insular species. Contr. Sci. 425: 1-12.

Haig, J., T.S. Hopkins \& T.B. Scanland. 1970. The shallow water anomuran crab fauna of southwestern Baja California, Mexico. Trans. San Diego Soc. Nat. Hist. 16: 13-32.

Harvey A.W. \& P.A. McLaughlin. 1991. Two new hermit crabs of the genus Pagurus (provenzanoi group) (Decapoda, Anomura, Paguridae) from the eastern Pacific, with notes on their ecology. Contr. Sci. 425: 13-21.

Hendrickx, M.E. 1992. Distribution and zoogeographic affinities of decapod crustaceans of the Gulf of California, Mexico. Proc. San Diego Soc. Nat. Hist. 20: 1-12.

Hendrickx, M.E. 1993. Crustáceos decápodos del Pacífico Mexicano. p. 271-318. In S.I. Salazar-Vallejo \& N.E.
González (eds.). Biodiversidad Marina y Costera de México. Comisión Nacional de Biodiversidad/Centro de Investigaciones Quintana Roo, Ciudad de México.

Hendrickx, M.E. 1995. Introducción. p. 1-7. In Fischer, W., F. Krupp, W. Schneider, C. Sommer, K.E. Carpenter y V.H. Niem. (eds.). Guía FAO para la identificación de especies para los fines de la pesca. Pacífico centro-oriental. Vol. I. Plantas e invertebrados. FAO, Roma.

Hernández, A.M.C. 1995. Taxonomía y distribución de la familia Porcellanidae (Crustacea: Decapoda: Anomura) del Pacífico Mexicano. Tesis de Licenciatura Fac. Cienc., Universidad Nacional Autónoma México, Ciudad de México.

McLaughlin, P.A. \& J. Haig. 1993. Two new species of the Pacific component of the Provenzanoi group of $\mathrm{Pa}$ gurus (Decapoda; Anomura; Paguridae) and a key to the regional species. Bull. Mar. Sci. 52: 642-668.

Markham, J.C., F.E. Donath-Hernández, J.L. Villalobos-Hiriart \& A. Cantú. 1990. Notes on the shallow-water marine Crustacea of the Caribbean coast of Quintana Roo, Mexico. An. Inst. Biol. UNAM 61: 405-446.

Moran, D.A. \& A.I Dittel. 1993. Anomuran and brachyuran crabs of Costa Rica: annotated list of species. Rev. Biol. Trop. 41: 599-617.

Steinbeck, J. \& E.F. Ricketts. 1941. Sea of Cortez. A Leisurely Journal of Travel and Research. Viking, New York.

Werding, B. \& J. Haig. 1983. The porcellanid crabs of the Isla Gorgona, Pacific Coast of Colombia, with a description of Clastotoechus gorgonensis sp. nov. (Crustacea: Anomura). An. Inst. Inv. Mar. Punta Betin 12: 57-70. 